\title{
BINARY ASTEROIDS: SECULAR PERTURBATIONS
}

\author{
R. VILIIENA DE MORAES and S. M. (i. WINTER \\ Depto. de Mecânica do Vòo e Orbital \\ Instituto Tecnológico de Aeronáutica \\ Centro Técnico Aeroespacial \\ 12225, São José dos Campos, SP, Brasil
}

\begin{abstract}
The motion of two small bodies orbiting each other whose barycenter is orbiting around a massive body is studied.The equations of motion are integrated considering the secular part of the disturbing function.
\end{abstract}

\section{Introduction}

Up to now a question without definitive answer is if there is or not double or multiple systems amidst the catalogued asteroids. A thorough analysis about this subject can be found in Chauvineau and Mignard (1990). The possibility of such objects to exist has been the incentive for the publication of several papers about the $2+2$ body problem, from which binary asteroids is an application (Whipple and Szebehely (1984), Milani and Nobili(1988)).

In this work is studied the motion of two hypothetical asteroids of masses $\boldsymbol{m}_{\mathbf{0}}$ and $m_{1}$, orbiting each other, whose barycenter is in keplerian orbit around the Sun, $m_{2}$. The perturbation of a fourth body (Jupiter, $m_{3}$ ) orbiting the primary is also considered.

\section{The Disturbing Function}

The equations of motion are referred to a system centered at $\operatorname{CM}\left(m_{0}, m_{1}\right)$, the center of mass of the binary system. It is assumed as known the motion of $m_{2}$ relative to $\operatorname{CM}\left(m_{0}, m_{1}\right)$ and the motion of $m_{3}$ relative to $m_{2}$, both motions taken as unperturbed elliptical motions.

Legendre polynomials, up to order 2, can be used to express the disturbing function in terms of the orbital elements. Neglecting the inclinations, the perturbations $F_{12}$ and $F_{13}$ of $m_{1}$ by $m_{2}$ and $m_{3}$ respectively, can be expressed in the following forms(Winter(1990):

$$
\begin{aligned}
F_{12}= & G m_{2}\left[\frac{a^{2}}{a^{\prime 3}}\left(A_{0}+e^{\prime} A_{1}+e^{\prime 2} A_{2}+e A_{3}+e^{2} A_{4}+e e^{\prime} A_{5}\right)\right], \\
F_{13}= & G m_{3}\left[\frac{a^{2}}{a_{23}^{3}}\left(B_{0}+e^{\prime} B_{1}+e^{\prime 2} B_{2}+e B_{3}+e^{2} B_{4}+e e^{\prime} B_{5}\right)\right. \\
& +\frac{a^{2} a^{\prime}}{a_{23}^{4}}\left(C_{0}+e^{\prime} C_{1}+e^{\prime 2} C_{2}+e C_{3}+e^{2} C_{4}+e e^{\prime} C_{5}\right) \\
+ & \left.\frac{a^{2} a^{\prime 2}}{a_{23}^{5}}\left(D_{0}+e^{\prime} D_{1}+e^{\prime 2} D_{2}+e D_{3}+e^{2} D_{4}+e e^{\prime} D_{5}\right)\right]
\end{aligned}
$$

where $A_{i}, B_{i}, C_{i}$, and $D_{i}(i=1, \ldots, 5)$ are functions of the eccentric anomalies, of the argument of pericenters and of the longitude of $m_{3}$. In those equations where 
considered only terms up to order 2 in the eccentricities and terms up to $a_{23}^{5}$ in the denominators. Moreover, $a_{23}$ represents the semi major axis of the orbit of $m_{3}$, unprimed orbital elements are used to indicate the orbital elements of $\boldsymbol{m}_{0}$ relative to $m_{1}$ and primes are used to represent the orbital elements of $m_{2}$.

\section{Secular Perturbations}

Using Bessel functions the equations of motion can be expressed in terms of the mean anomaly. Taking into account only the secular part $F$, of the disturbing function, the following equations are obtained from the Lagrange equations in nonsingular variables $h=e \sin \varpi, k=e \cos \varpi$ :

$$
\begin{gathered}
\dot{h}=\frac{1}{n a^{2}} \frac{\partial F_{s}}{\partial k}=(1,1) k, \\
\dot{k}=-\frac{1}{n a^{2}} \frac{\partial F_{g}}{\partial h}=-(1,1) h,
\end{gathered}
$$

where, considering terms up to the order of $e^{2}$,

$$
(1,1)=\frac{3 G}{4 n}\left[\frac{m_{2}}{a^{\prime 3}}+\frac{m_{3}}{a_{23}^{3}}\left(1-\frac{3 a^{\prime}}{a_{23}}\right)\right] .
$$

This system is easily integrable by analytical techniques. This result was applied to find the precessional motion of a hypothetical system of binary asteroids, and it is in accordance with the numerical experiments obtained by Pojman (1987).

\section{Conclusions}

An analytical solution was obtained to compute secular perturbations(planar case), due to the Sun and Jupiter, on binary asteroids. In general the perturbation due to Jupiter is about $10^{4}$ smaller.

\section{Acknowledgements}

This work was partially sponsored by FAPESP.

\section{References}

Chauvineau, B. and Mignard F. (1990) 'Dynamics of Binary Asteroids', Icarus, 83, 360-381.

Milani, A. and Nobili, A.M. (1988) 'Instability of The $2+2$ Body Problem', Celest. Mech., 41, 153-160.

Pojman, J.R. (1987)' Motion of Binary Asteroids', M.Sc.Thesis, The University of Texas, Austin.

Whipple, A.L. and Szebehely, V. (1984) 'The Restricted Problem of $n+\nu$ Bodies', Celest. Mech., 32, $137-144$.

Winter, S.M. (1990) ' Perturbações Seculares no Problema Restrito de $2+2$ Corpos', M.Sc. Thesis, ITA, São José dos Campos. 